\title{
Determinants of Integrated Management of Childhood Illness (IMCI) non-severe pneumonia classification and care in Malawi health facilities: Analysis of a national facility census
}

\author{
Emily White Johansson', \\ Humphreys Nsona², \\ Liliana Carvajal-Aguirre ${ }^{3}$, \\ Agbessi Amouzou ${ }^{3}$, \\ Helena Hildenwall ${ }^{4}$
${ }^{1}$ Department of Women's and Children's Health, Uppsala University, Uppsala, Sweden
2 Integrated Management of Childhood Illness (IMCI) Unit, Ministry of Health, Lilongwe, Malawi
${ }^{3}$ Data and Analytics Section, United Nations Children's Fund, New York, New York, USA
${ }^{4}$ Global Health - Health Systems and Policy Research Group, Department of Public Health Sciences, Karolinska Institutet, Stockholm, Sweden

Background Research shows inadequate Integrated Management of Childhood Illness (IMCI)-pneumonia care in various low-income settings but evidence is largely from small-scale studies with limited evidence of patient-, provider- and facility-levels determinants of IMCI non-severe pneumonia classification and its management.

Methods The Malawi Service Provision Assessment 2013-2014 included 3149 outpatients aged 2-59 months with completed observations, interviews and re-examinations. Mixed-effects logistic regression models quantified the influence of patient-, provider and facility-level determinants on having IMCI non-severe pneumonia and its management in observed consultations.

Findings Among 3149 eligible outpatients, 590 (18.7\%) had IMCI non-severe pneumonia classification in re-examination. 228 (38.7\%) classified cases received first-line antibiotics and 159 (26.9\%) received no antibiotics. $18.6 \%$ with cough or difficult breathing had 60-second respiratory rates counted during consultations, and conducting this assessment was significantly associated with IMCI training ever received (odds ratio $(O R)=2.37,95 \%$ confidence interval $(C I): 1.29-4.31$ ) and negative rapid diagnostic test results $(\mathrm{OR}=3.21,95 \% \mathrm{CI}: 1.45-7.13)$. Older children had lower odds of assessments than infants ( $O R=48-59$ months: $0.35,95 \% \mathrm{CI}: 0.16-0.75)$. Children presenting with any of the following complaints also had reduced odds of assessment: fever, diarrhea, skin problem or any danger sign. First-line antibiotic treatment for classified cases was significantly associated with high temperatures $(\mathrm{OR}=3.26,95 \% \mathrm{CI}: 1.24-8.55)$ while older children had reduced odds of first-line treatment compared to infants ( $\mathrm{OR}=48-59$ months: 0.29 , 95\% CI: 0.10-0.83). RDT-confirmed malaria was a significant predictor of no antibiotic receipt for IMCI non-severe pneumonia $(\mathrm{OR}=10.65$, 95\% CI: 2.39-47.36).

\section{Correspondence to:}

Emily White Johansson, PhD Uppsala University

Department of Women's and Children's Health

International Maternal and Child Health Akademiska Sjukhuset

SE-751 85 Uppsala, Sweden

emily.johansson@kbh.uu.se
Conclusions IMCI non-severe pneumonia care was sub-optimal in Malawi health facilities in 2013-2014 with inadequate assessments and prescribing practices that must be addressed to reduce this leading cause of mortality. Child's symptoms and age, malaria diagnosis and provider training were primary influences on assessment and treatment practices. Current evidence could be used to better target IMCI training and support to improve pneumonia care for sick children in Malawi facilities. 
Despite the enormous progress in child survival over the past two decades, approximately six million children under five years still die each year globally [1]. These deaths largely occur during the neonatal period or are due to infectious causes, such as pneumonia [2]. Indeed, pneumonia remains a leading cause of child mortality accounting for nearly one million under-five deaths annually. Early and effective treatment of childhood pneumonia is therefore a cornerstone of child survival programs [3].

Since the 1990s, WHO and UNICEF have promoted the Integrated Management of Childhood Illness (IMCI) strategy in low- and middle-income countries to effectively manage pneumonia and other common causes of child morbidity and mortality in an integrated manner [4]. While IMCI has great potential to improve health worker performance and quality care for sick children [5], poor implementation in routine practice has been documented in various settings over the past few decades [6,7]. This includes inadequate care for the IMCI-pneumonia algorithm in particular, which has been demonstrated in Malawi $[8,9]$ although a more recent study from rural Malawi suggested stronger IMCI-pneumonia performance among service providers [10].

Yet this evidence is largely derived from small-scale studies in limited facility contexts without examination of determinants of IMCI-pneumonia classification or its case management. A national facility census, or Service Provision Assessment (SPA), was conducted in Malawi in 2013-2014 that included observed sick child consultations and re-examinations [11]. While an analysis of overall correct IMCI-pneumonia care is not possible using this facility census given limited observation and re-examination protocols (see Methods), it does provide a unique opportunity to identify determinants of having an IMCI non-severe pneumonia episode, such as child's age or symptoms [12,13]. It also allows for a wide ranging assessment of patient-, provider- and facility-level predictors for conducting select assessments (60-second respiratory rate count) and prescribing first-line treatment to classified cases. Such evidence could help target IMCI training and support going forward in order to improve quality pneumonia care across Malawi health facilities.

\section{METHODS}

\section{Study setting}

Malawi is a low-income country in sub-Saharan Africa with an estimated population of 17 million [14]. Malawi's under-five mortality rate declined from 242 deaths per 1000 live births in 1990 to 64 in 2015 [1], which achieved the Millennium Development Goal for child mortality. This significant reduction has been attributed to scaling-up interventions effective against the leading causes of child death, reducing child undernutrition and mother-to-child HIV transmission as well as improving quality childbirth care [15]. Prior to the Malawi Service Provision Assessment (SPA) in 2013-2014, IMCI guidelines were last updated in 2013 to reflect test-based malaria case management and wide-scale IMCI in-service training was previously implemented in 2009. Nationwide deployment of malaria rapid diagnostic tests (RDT) was initiated in July 2011 accompanied by training in RDT safety and use along with basic information on managing RDT-negative cases [16].

The Malawi health system generally includes both government facilities and publicly-supported facilities managed by the Christian Health Association of Malawi (CHAM) [16]. The three facility levels include health centers, district hospitals and regional hospitals. Health centers are the lowest level and deliver primary health care services that are generally led by a medical assistant or nurse midwife technician. District hospitals are referral facilities at the next level that provide in-patient care, laboratory diagnostics and maternity care that are generally led by medical doctors and clinical officers. Regional or central hospitals are the highest level that are generally research and teaching institutions that provide specialized medical care. Community treatment services are also available for sick children but were not included in this facility-based assessment.

\section{Survey methods}

The Malawi Service Provision Assessment (SPA) was conducted in June 2013-February 2014 by the Ministry of Health and The DHS Program, which includes facility and laboratory audits, observed consultations with limited re-examination, patient exit interviews and health worker interviews. Survey methods are described elsewhere including procedures for obtaining ethical approval and participant consent [11].

Briefly, Malawi SPA 2013-2014 was designed as a census of all formal public and private facilities in the country to include 977 facilities out of 1060 on the Ministry of Health master facility list. Non-response 
was due to refusal (3\%), closure (2\%), inaccessibility (2\%) or other issue (1\%). At each facility, outpatients were systematically selected for observation based on the expected patient load for sick child curative services on the interview date in order to yield no more than 15 observations per facility. Outpatients were eligible to participate if they were less than five years old and presented with an illness complaint that was not an exclusive injury or non-disease condition. Children aged 2-59 months attending an observed outpatient consultation were included in this study if consent for the observed consultation, exit interview and re-examination were obtained. A total of 3149 observations met these criteria and were included in the analysis (Figure 1). A median of 3 observations were conducted at each facility.

During each observed sick child consultation, an observer was present to silently record whether certain IMCI assessments or examinations were completed, such as felt the child for fever or body hotness, counted breathing for 60 seconds or checked skin turgor for dehydration. However, there is no recording of assessment quality nor were all IMCI assessments included in the observer checklist, notably it was not recorded if the provider checked for chest in-drawing or asked about illness duration. After each consultation, the observer asked the provider to report all diagnoses/classifications and treatments prescribed to the sick child. A limited re-examination protocol was conducted during the exit interview and included measuring temperature, checking anemia symptoms and counting respiratory rates for 60 seconds if cough or difficult breathing (CDB) was reported. Both the observed consultation and re-examination was conducted by clinicians, nurses or nurse midwives trained in these specific protocols. For these reasons, it was not possible to directly assess correct pneumonia case management by comparing observed practices with 'gold standard' re-examination since neither the observation or re-examination provided complete information for such analyses. Nevertheless, the large number of facilities audited and broad data collection scope provide an opportunity to examine a wide-range of determinants for having a IMCI non-severe pneumonia episode, performing certain assessments and prescribing antibiotics for classified cases.

\section{Classification of IMCI non-severe pneumonia}

IMCI non-severe pneumonia was defined in this study according to 2013 Malawi IMCI guidelines [17]. It was based on re-examination of the child as having a cough and difficult breathing (CDB) complaint and rapid breathing of 50 or more breaths per minute ( 2 up to 12 months) or 40 or more breaths per minute (12 to 59 months). IMCI severe pneumonia is classified based on chest in-drawing with or without fast breathing. However, chest in-drawing assessment was not part of re-examination or recorded as

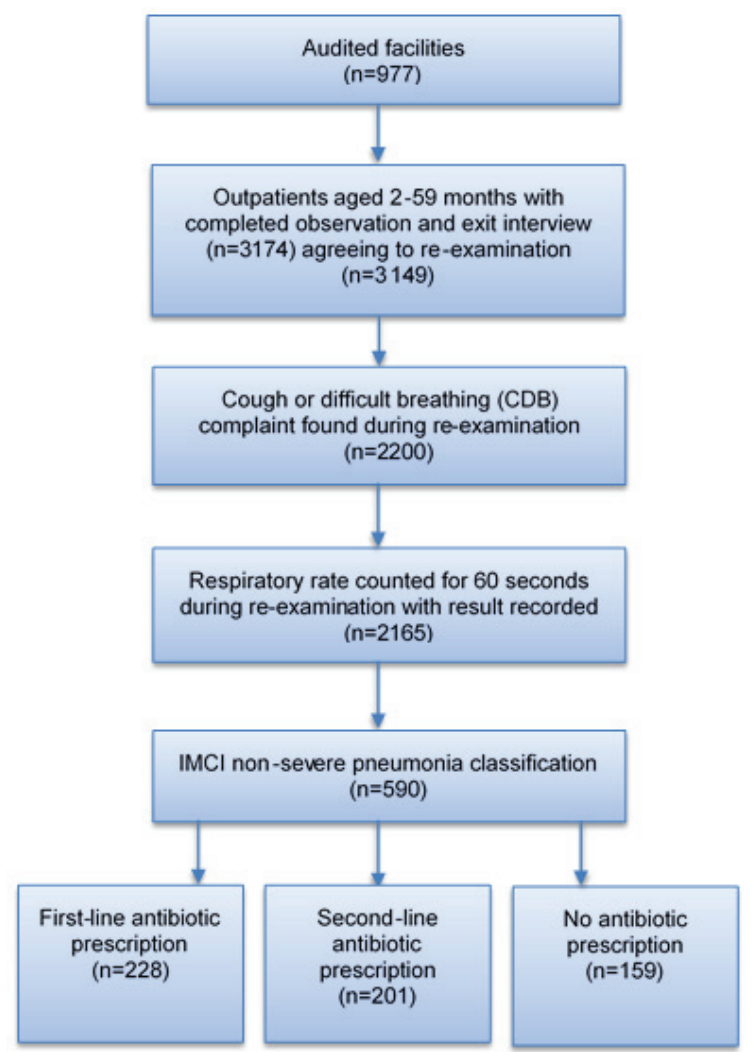

Figure 1. Study sample. Frequencies were weighted to account for the unequal probabilities of selection due to differing client volumes on the interview date. IMCI non-severe pneumonia classification is defined as cough or difficult breathing complaint and a respiratory rate of 50 breaths or more per minute ( 2 up to 12 months) or 40 breaths or more per minute (12 months up to 5 years) recorded during re-examination. First-line antibiotic prescription refers to benzyl penicillin injection or amoxicillin (capsule or syrup). Second-line antibiotic prescription refers to cotrimoxazole (syrup or tablet) or other antibiotic (injection, syrup or capsule). Antibiotic treatment totals sum to 588 observations (not 590) due to missing values. 
done in the observed consultation. For this reason, our analysis focuses on IMCI non-severe pneumonia since a raised respiratory rate at least indicates non-severe classification. In 2014, IMCI guidelines were updated to define non-severe pneumonia as fast breathing and/or chest in-drawing but this revision occurred after survey implementation and is not used in this paper [18].

\section{Assessment of IMCI non-severe pneumonia}

IMCI non-severe pneumonia assessment was defined as a 60-second respiratory rate count observed and recorded during the consultation. This analysis was based on the subset of outpatients aged 2-59 months with CDB complaint reported in the exit interview.

\section{Treatment of IMCI non-severe pneumonia}

IMCI non-severe pneumonia treatment was derived from provider reports of treatments prescribed to the sick child after the observation. First-line antibiotic treatment included benzyl penicillin injection or amoxicillin (capsule or syrup) since it was not possible to differentiate severe and non-severe cases in this data set. Second-line antibiotic treatment included cotrimoxazole (syrup or tablet) or other antibiotic (injection, syrup or capsule). Hierarchical coding assigned the most appropriate prescription to the observation if multiple drugs were prescribed. This analysis was based on the subset of outpatients aged 2-59 months with IMCI non-severe pneumonia that attended a facility with amoxicillin available on the interview date. Only 13 IMCI non-severe pneumonia cases (unweighted) were observed in facilities without amoxicillin and these observations were removed from this analysis.

\section{Explanatory variables}

Patient-, provider and facility-level variables with potential to affect IMCI-pneumonia classification and care were explored in these analyses [8-10,19-25]. Patient-level variables included child's sex and age (2-11, 12-23, 24-35, 36-47, 48-59 months), caregiver's age (Under 20, 20-29, 30-39, 40 or more years), caregiver's education (none, primary or at least secondary attendance), RDT conducted prior to or during the initial consultation according to provider reports, and if so, reported RDT results (positive or negative), child's temperature (37.5 or less, 37.6-38.9 or 39.0-40.8) recorded in re-examination, illness duration (0-1, 2-4, 5 or more days), consultation start hour (7-10 am, 11-2 pm, 3-5 pm) recorded in the observation, reported wait time (under $10,11-30,31-60,60$ or more minutes), first or follow-up visit for the current illness, and symptom complaints (fever, diarrhea, ear problem, eye problem, skin problem or any danger sign). Any danger sign includes vomits everything, convulsions, lethargy or inability to eat, drink or breastfeed. Patient-level variables were reported by the caregiver during the exit interview unless otherwise noted.

Provider-level variables included job qualification (doctor, medical assistant, nurse or other provider) and year qualification received (before 2000, 2001-2009, 2010 to present), provider sex, supervisor or incharge status, IMCI in-service training ever received or not and recent supervisory visit (none, within past 3 months, over 3 months ago). Provider-level variables were reported during the provider interview.

Facility-level variables included facility type (hospital or other), managing authority (government or CHAM/other private), total staff doctors ( $0,1,2+$ doctors), routine general user fees or not, routine management meetings reportedly occur or not, region (north, central, south), location (urban, rural), timer available, amoxicillin or any antibiotic available, as well as malaria risk (infection prevalence) values for 2013-2014 linked to data sets through geocoded facility locations and transmission season estimates derived from facility locations and interview date [26,27]. Facility-level variables were recorded during the facility audit unless otherwise noted.

\section{Missing values}

Observations with missing values were removed from analyses using listwise deletion. For the IMCI nonsevere pneumonia classification analysis, 158 (5\%) observations were dropped that had missing values for at least one explanatory variable. For the assessment and treatment analyses, 65 (3\%) and 9 (2\%) observations were removed respectively.

\section{Data analyses}

Mixed-effects logistic regression models quantified the influence of explanatory variables on the binary outcomes of interest. Variables were included as categorical fixed effects nested within facility identifiers 
and normal distribution of the random effects was assumed. Bivariate analyses were initially conducted for each variable to estimate crude odds ratios separately for each of the outcomes. Variables found to be statistically significant at the 0.1 level in bivariate analyses were subsequently included in final models to obtain adjusted odds ratios. Variance inflation factors were used to detect multi-collinearity among variables prior to inclusion in final models. Given the importance of child's age in model outputs, we tested for an interaction between child's age and IMCI training on the assessment and treatment outcomes in final models. Results were stratified to examine effect differences across age groups (2-11, 12-23, 24-35, 36-47, 48-59 months). Point estimates were calculated using weights to account for unequal probabilities of selection due to differing client volumes at facilities on the interview date. Standard error estimation accounted for clustering of client observations within facilities. The level of statistical significance was set to 0.05. Stata 13.1 (Stata Corp., College Station, TX) was used for analyses.

\section{RESULTS}

Among 3149 eligible outpatients aged 2-59 months, 590 (18.7\%) were classified with IMCI non-severe pneumonia in re-examination (Table 1). Among these classified cases, 228 (38.7\%) received benzyl pen-

Table 1. Characteristics of outpatients aged 2-59 months with IMCI non-severe pneumonia, Malawi health facilities, 2013-2014*

\begin{tabular}{|c|c|c|c|c|}
\hline & & $\begin{array}{l}\text { OUtPatients aGed } \\
\text { 2-59 months ( } \mathbb{N l o .} \text {.) }\end{array}$ & $\begin{array}{l}\text { IMCI NON-SEVERE } \\
\text { PMEUMONIA (o.) }\end{array}$ & $\begin{array}{l}\text { Percent IMCI NON-SEVERE } \\
\text { PNEUMONIA (95\% CI) }\end{array}$ \\
\hline Total & & 3149 & 590 & $18.7(16.6-21.1)$ \\
\hline \multirow[t]{2}{*}{ Fever complaint } & Yes & 2110 & 397 & $18.8(16.4-21.5)$ \\
\hline & No & 962 & 164 & $17.0(13.5-21.3)$ \\
\hline \multirow[t]{2}{*}{ Diarrhea complaint } & Yes & 899 & 166 & $18.5(15.2-22.3)$ \\
\hline & No & 2249 & 423 & $18.7(16.3-21.5)$ \\
\hline \multirow[t]{2}{*}{ Danger sign complaint } & Yes & 1481 & 283 & $19.1(16.3-22.4)$ \\
\hline & No & 1669 & 307 & $18.4(15.7-21.4)$ \\
\hline \multirow[t]{2}{*}{ RDT result } & Positive & 378 & 78 & $20.6(15.1-27.5)$ \\
\hline & Negative & 653 & 151 & $23.2(18.8-28.1)$ \\
\hline \multirow[t]{3}{*}{ Temperature (Celsius) } & 37.5 or less & 2436 & 410 & $16.8(14.5-19.3)$ \\
\hline & $37.6-38.9$ & 595 & 144 & $24.3(19.9-29.2)$ \\
\hline & $39.0-40.8$ & 99 & 30 & $30.6(21.7-41.3)$ \\
\hline \multirow{5}{*}{ Child's age (months) } & $2-11$ & 1124 & 166 & $14.7(11.5-18.7)$ \\
\hline & $12-23$ & 912 & 261 & $28.6(24.6-33.0)$ \\
\hline & $24-35$ & 540 & 88 & $16.2(12.6-20.7)$ \\
\hline & $36-47$ & 317 & 39 & $12.2(8.4-17.3)$ \\
\hline & $48-59$ & 257 & 37 & $14.5(9.6-21.2)$ \\
\hline \multirow[t]{3}{*}{ Illness duration (days) } & $0-1$ & 726 & 135 & $18.6(15.0-22.7)$ \\
\hline & $2-4$ & 1992 & 363 & $18.2(15.7-21.0)$ \\
\hline & 5 or more & 420 & 92 & $22.0(17.0-28.0)$ \\
\hline \multirow[t]{2}{*}{ Malaria endemicity $\left(\mathrm{PfPR}_{2-10}\right)$} & Under 0.20 & 2367 & 448 & $18.9(16.4-21.6)$ \\
\hline & $0.20-0.39$ & 782 & 142 & $18.2(14.4-22.7)$ \\
\hline \multirow[t]{2}{*}{ Transmission season } & Peak & 428 & 68 & $15.9(12.3-20.3)$ \\
\hline & Off-peak & 2721 & 522 & $19.2(16.8-21.8)$ \\
\hline \multirow[t]{2}{*}{ Residence } & Urban & 1007 & 170 & $16.8(12.4-22.5)$ \\
\hline & Rural & 2142 & 420 & $19.6(17.5-22.0)$ \\
\hline \multirow[t]{3}{*}{ Region } & North & 463 & 68 & $14.7(11.5-18.7)$ \\
\hline & Central & 1583 & 317 & $20.0(17.2-23.1)$ \\
\hline & South & 1103 & 205 & $18.4(14.6-23.4)$ \\
\hline \multirow[t]{2}{*}{ Any antibiotic observed } & Yes & 3142 & 589 & $18.6(16.5-20.9)$ \\
\hline & No & 3 & $<1$ & $25.0(-)$ \\
\hline \multirow[t]{2}{*}{ Amoxicillin observed } & Yes & 3094 & 580 & $18.7(16.6-21.1)$ \\
\hline & No & 52 & 10 & $18.8(9.9-33.0)$ \\
\hline \multirow[t]{2}{*}{ Facility type } & Hospital (central, district, rural, other) & 1136 & 200 & $17.6(13.1-23.2)$ \\
\hline & Other facility type & 2014 & 390 & $19.4(17.4-21.5)$ \\
\hline \multirow[t]{2}{*}{ Managing authority } & Government & 2404 & 454 & $18.9(16.3-21.8)$ \\
\hline & CHAM or other private ownership & 745 & 136 & $18.2(15.2-21.8)$ \\
\hline
\end{tabular}

IMCI - Integrated Management of Childhood Illness, CI - confidence interval, RDT - rapid diagnostic test, PfPR - Plasmodium falciparum parasite rate, CHAM - Christian Health Association of Malawi

* Outpatients aged 2-59 months with completed observations, exit interviews and re-examinations were included. IMCI non-severe pneumonia classification was identified in re-examination based on CDB complaint and a 60-second respiratory rate count of 50 or more breaths per minute ( 2 up to 12 months) and 40 or more breaths per minute ( 12 months to 5 years). Frequencies and cross-tabulations were weighted to account for the unequal probabilities of selection due to differing client volumes on the interview date. 
icillin injection or amoxicillin, 157 (26.6\%) received cotrimoxazole, 44 (7.5\%) received other antibiotics and 159 (26.9\%) received no antibiotic (Figure 1). Among 2271 outpatients aged 2-59 months with CDB complaints reported in the exit interview, $422(18.6 \%)$ had a 60-second respiratory rate counted in the consultation (Online Supplementary Document).

\section{IMCI non-severe pneumonia classification}

Table 2 indicates a significant association between child's age, raised temperature and illness duration on having IMCI non-severe pneumonia classification. Compared to infants, there was nearly three times higher odds of having this classification among 12-23 months (odds ratio $(\mathrm{OR})=2.87,95 \%$ confidence interval (CI): 2.17-3.78) while there was no statistical difference with older age groups. Outpatients with a moderate or high temperature had 1.59 (95\% CI: 1.21-2.09) and 2.38 (95\% CI: 1.41-4.04) times higher odds of receiving this classification respectively than those with no or low temperature. Compared to a short illness duration ( $0-1$ day), outpatients reporting illness lasting 5+ days had 1.57 (95\% CI: 1.082.27) times higher odds of having IMCI non-severe pneumonia.

\section{Count respiratory rates for 60 seconds}

Table 3 shows the significance of child's age, symptoms, IMCI in-service training, provider qualification, region and reported wait time on the odds of receiving a 60-second respiratory rate count to assess IMCI non-severe pneumonia. There was a significant and consistent decline in assessment odds with increas-

Table 2. Determinants of IMCI non-severe pneumonia classification in outpatients aged 2-59 months, Malawi health facilities, 2013-2014*

\begin{tabular}{|c|c|c|c|c|c|}
\hline & & & Adjusted OR & $95 \%$ CI & $\mathbf{P}$ \\
\hline \multirow[t]{17}{*}{ Patient } & Fever complaint & No & 1.00 & & \\
\hline & & Yes & 1.07 & $0.83-1.38$ & 0.616 \\
\hline & Diarrhea complaint & No & 1.00 & & \\
\hline & & Yes & 0.80 & $0.62-1.03$ & 0.090 \\
\hline & Danger sign complaint & No & 1.00 & & \\
\hline & & Yes & 1.09 & $0.87-1.37$ & 0.438 \\
\hline & Temperature (Celsius) & 37.5 or less & 1.00 & & \\
\hline & & $37.6-38.9$ & 1.59 & $1.21-2.09$ & 0.001 \\
\hline & & $39.0-40.8$ & 2.38 & $1.41-4.04$ & 0.001 \\
\hline & Child's age (months) & $2-11$ & 1.00 & & \\
\hline & & $12-23$ & 2.87 & $2.17-3.78$ & $<0.001$ \\
\hline & & $24-35$ & 1.25 & $0.89-1.76$ & 0.192 \\
\hline & & $36-47$ & 0.94 & $0.62-1.45$ & 0.794 \\
\hline & & $48-59$ & 1.05 & $0.67-1.66$ & 0.824 \\
\hline & Illness duration (days) & $0-1$ & 1.00 & & \\
\hline & & $2-4$ & 1.08 & $0.82-1.43$ & 0.571 \\
\hline & & 5 or more & 1.57 & $1.08-2.27$ & 0.016 \\
\hline \multirow[t]{13}{*}{ Facility } & Malaria endemicity $\left(\mathrm{PfPR}_{2-10}\right)$ & Less than 0.20 & 1.00 & & \\
\hline & & $0.20-0.39$ & 0.76 & $0.52-1.10$ & 0.148 \\
\hline & Transmission season & Peak & 1.00 & & \\
\hline & & Off-peak & 0.89 & $0.61-1.29$ & 0.538 \\
\hline & Residence & Urban & 1.00 & & \\
\hline & & Rural & 1.26 & $0.87-1.82$ & 0.225 \\
\hline & Region & North & 1.00 & & \\
\hline & & Central & 1.48 & $0.98-2.24$ & 0.061 \\
\hline & & South & 0.91 & $0.61-1.35$ & 0.623 \\
\hline & Facility type & Hospital (central, district, rural, other) & 1.00 & & \\
\hline & & Other facility type & 1.06 & $0.71-1.59$ & 0.769 \\
\hline & Managing authority & Government & 1.00 & & \\
\hline & & CHAM or other private ownership & 0.93 & $0.69-1.26$ & 0.654 \\
\hline
\end{tabular}

IMCI - Integrated Management of Childhood Illness, CI - confidence interval, OR - odds ratio, PfPR - Plasmodium falciparum parasite rate, CHAM - Christian Health Association of Malawi

*Variables presented in this table were significant $(P<0.1)$ in bivariate analyses and were then included simultaneously in the final model to obtain adjusted odds ratios. Mixed-effects logistic regression models quantified the influence of the above variables on receiving IMCI non-severe pneumonia classification (or not) adjusted for data clustering. 
Table 3. Determinants of taking a 60-second respiratory rate count in outpatients aged 2-59 months with cough or difficult breathing complaints, Malawi health facilities, 2013-2014*

\begin{tabular}{|c|c|c|c|c|c|}
\hline & & & AdJusted OR & $95 \%$ CI & $\mathbf{P}$ \\
\hline \multirow[t]{23}{*}{ Patient } & Fever complaint & No & 1.00 & & \\
\hline & & Yes & 0.62 & $0.43-0.95$ & 0.018 \\
\hline & Diarrhea complaint & No & 1.00 & & \\
\hline & & Yes & 0.61 & $0.43-1.01$ & 0.023 \\
\hline & Skin problem complaint & No & 1.00 & & \\
\hline & & Yes & 0.21 & $0.07-0.64$ & 0.006 \\
\hline & Any danger sign complaint & No & 1.00 & & \\
\hline & & Yes & 0.70 & $0.49-1.02$ & 0.052 \\
\hline & Child's age (months) & $2-11$ & 1.00 & & \\
\hline & & $12-23$ & 0.62 & $0.49-0.95$ & 0.030 \\
\hline & & $24-35$ & 0.38 & $0.22-0.65$ & $<0.001$ \\
\hline & & $36-47$ & 0.53 & $0.27-1.05$ & 0.070 \\
\hline & & $48-59$ & 0.35 & $0.16-0.75$ & 0.007 \\
\hline & Caregiver's age (years) & $11-19$ & 1.00 & & \\
\hline & & $20-29$ & 0.64 & $0.35-1.14$ & 0.125 \\
\hline & & $30-39$ & 1.20 & $0.61-2.27$ & 0.627 \\
\hline & & 40 or older & 0.33 & $0.10-1.14$ & 0.080 \\
\hline & Wait time (minutes) & 10 or less & 1.00 & & \\
\hline & & $11-30$ & 0.42 & $0.22-0.81$ & 0.009 \\
\hline & & $31-59$ & 0.74 & $0.41-1.32$ & 0.303 \\
\hline & & 60 or more & 1.00 & $0.56-1.75$ & 0.955 \\
\hline & RDT results $\dagger$ & Positive & 1.00 & & \\
\hline & & Negative & 3.21 & $1.45-7.13$ & 0.001 \\
\hline \multirow[t]{10}{*}{ Facility } & Malaria endemicity $\left(\mathrm{PfPR}_{2-10}\right)$ & Less than 0.20 & 1.00 & & \\
\hline & & $0.20-0.39$ & 1.25 & $0.60-2.61$ & 0.630 \\
\hline & Region & North & 1.00 & & \\
\hline & & Central & 1.15 & $0.50-2.60$ & 0.749 \\
\hline & & South & 0.31 & $0.13-0.71$ & 0.006 \\
\hline & Doctors (total on staff) & 0 & 1.00 & & \\
\hline & & 1 & 1.40 & $0.38-5.49$ & 0.593 \\
\hline & & 2 or more & 0.33 & $0.09-1.17$ & 0.085 \\
\hline & IMCI guidelines available & No & 1.00 & & \\
\hline & & Yes & 1.19 & $0.64-2.32$ & 0.546 \\
\hline \multirow[t]{10}{*}{ Provider } & Qualification & Doctor & 1.00 & & \\
\hline & & Medical assistant & 0.78 & $0.37-1.80$ & 0.607 \\
\hline & & Nurse or other provider & 0.33 & $0.12-1.01$ & 0.051 \\
\hline & Supervisor or in-charge & No & 1.00 & & \\
\hline & & Yes & 1.29 & $0.68-2.46$ & 0.425 \\
\hline & Qualification received (year) & Before 2000 & 1.00 & & \\
\hline & & $2000-2009$ & 1.15 & $0.56-2.33$ & 0.713 \\
\hline & & 2010 to present & 0.69 & $0.30-1.60$ & 0.394 \\
\hline & $\begin{array}{c}\text { IMCI in-service training (ever } \\
\text { received) }\end{array}$ & No & 1.00 & & \\
\hline & & Yes & 2.37 & $1.29-4.31$ & 0.006 \\
\hline
\end{tabular}

CI - confidence interval, OR - odds ratio, RDT - rapid diagnostic test, PfPR - Plasmodium falciparum parasite rate, CHAM - Christian Health Association of Malawi, IMCI - Integrated Management of Childhood Illness

*Table S1 in Online Supplementary Document presents descriptive statistics for outpatients aged 2-59 months with CDB complaints reported in exit interviews $(n=2271)$. CI refers to confidence interval. Variables presented in this table were significant $(P<0.1)$ in bivariate analyses and were then included simultaneously in the final model to obtain adjusted odds ratios. Mixed-effects logistic regression models quantified the influence of the above variables on counting respiratory rates for $60 \mathrm{~s}$ (or not) adjusted for data clustering.

$\uparrow R D T$ results is based on a subset analysis of outpatients with CDB complaints and reported RDT results $(\mathrm{n}=692)$.

ing age of the child. Compared to infants, the odds of receiving a 60-second respiratory rate count declined by $38 \%$ for $12-23$ months ( $O R=0.62,95 \%$ CI: $0.49-0.95$ ), by $62 \%$ for $24-35$ months (OR $=0.38$, 95\% CI: $0.22-0.65)$, by $47 \%$ for $36-47$ months (OR $=0.53,95 \%$ CI: $0.27-1.05$ ) and by $65 \%$ for $48-59$ months (OR=0.35, 95\% CI: 0.16-0.75).

Reporting other symptoms with CDB complaint significantly reduced the odds of having a 60-second respiratory rate counted in the consultation. Assessment odds declined by $38 \%$ if fever was reported $(\mathrm{OR}=0.62,95 \% \mathrm{CI}: 0.43-0.95)$ compared to not reported, by 39\% if diarrhea was reported (OR=0.61, 
95\% CI: $0.43-1.01$ ), by $79 \%$ for reported skin problems (OR=0.21, $95 \%$ CI: $0.07-0.64$ ), and by $30 \%$ if any danger sign was reported although the latter was not a statistically significant reduction $(\mathrm{OR}=0.70$, 95\% CI: 0.49-1.02).

Outpatients attended by providers that ever received IMCI in-service training had 2.37 times higher odds of receiving a 60-second respiratory rate count (95\% CI: 1.29-4.31) than those seen by providers without such training. Compared to doctors, clients attended by nurses, midwives or other lower-level providers had reduced assessment odds (OR=0.33, 95\% CI: 0.12-1.01) although there was no significant difference with medical assistants. Attendance at facilities in the South region was associated with significantly lower assessment odds (OR=0.31, 95\% CI: 0.13-0.71) than in the North region. In a subset analysis of outpatients with CDB complaints and reported RDT results $(n=692)$, RDT-negative cases had 3.21 times higher assessment odds than outpatients with RDT-confirmed malaria (95\% CI: 1.45-7.13) in the adjusted analysis.

There was also evidence of an interaction between categorical variables child's age and IMCI training ( $\mathrm{p}-$ values ranged from 0.006 to 0.813 ) on the assessment outcome. To further explore this result, the final model was stratified by age groupings. Outpatients aged $12-23$ months (OR=9.56, 95\% CI: 3.03-30.18) and 48-59 months (OR=261.97, 95\% CI: 1.46-47281.50) that visited providers ever receiving IMCI in-service training had significantly higher assessment odds than outpatients seen by providers with no such training although effect sizes should be interpreted with caution due to few observations and positive outcomes (Table 4). There was a negligible difference between those visiting trained and untrained providers in other age groups. There was also limited overlap in confidence intervals of the adjusted odds ratios for 2-11 and 12 to 23-month-olds suggesting a difference across these age groups in the effect of training on assessment odds. Stratified models for the treatment outcome showed no significant difference in IMCI training across age groupings (data not shown).

\section{Antibiotic prescriptions}

Figure 2 depicts antibiotic prescriptions for IMCI non-severe pneumonia cases across different age groups. Nearly half (45.9\%) of infants with IMCI non-severe pneumonia received first-line antibiotics and this proportion consistently declined with older ages to a low of $18.3 \%$ among 48 to $59-$ month-olds. In contrast, second-line antibiotics (cotrimoxazole or other antibiotic) were more often prescribed to older children with $50.5 \%$ of 48 to $59-$ month-olds incorrectly treated compared to $26.2 \%$ of infants. The proportion of outpatients receiving no antibiotic for IMCI non-severe pneumonia ranged from $22.0 \%$ among 24 to $35-$ month-olds to $31.2 \%$ among those $48-59$ months old.

First-line antibiotic treatment of classified cases was significantly associated with younger ages, high temperatures and more staff doctors (Table 5). Compared to infants, odds of first-line treatment declined by $67 \%$ for ages $36-47$ months (OR=0.33, 95\% CI: $0.12-0.88$ ) and by $71 \%$ for ages $48-59$ months $(\mathrm{OR}=0.29$, 95\% CI: 0.10-0.83) while there was no statistical difference with other age groups. Outpatients with high temperatures $\left(39.0^{\circ} \mathrm{C}\right.$ or more) had 3.26 times higher odds of receiving first-line antibiotics than those with low or no temperature (95\% CI: 1.24-8.55). Children with IMCI non-severe pneumonia attending facilities with two or more staff doctors had 2.90 times higher odds of receiving first-line antibiotic treatment than those visiting facilities with no staff doctors (95\% CI: 1.22-6.88).

Table 4. Effect of IMCI training on counting respiratory rates for $60 \mathrm{~s}$ across age groupings, Malawi health facilities, 2013-2014*

\begin{tabular}{|c|c|c|c|c|}
\hline & IMCI IN-SERVICE tRAINING (EVER RECEIVED OR NOT) & Adjusted OR & $95 \%$ CI & $\mathbf{P}$ \\
\hline \multirow[t]{2}{*}{ 2-11 months } & No & 1.00 & & \\
\hline & Yes & 1.50 & $0.70-3.24$ & 0.301 \\
\hline \multirow[t]{2}{*}{$12-23$ months } & No & 1.00 & & \\
\hline & Yes & 9.56 & $3.03-30.18$ & $<0.001$ \\
\hline \multirow[t]{2}{*}{$24-35$ months } & No & 1.00 & & \\
\hline & Yes & 2.06 & $0.60-7.07$ & 0.250 \\
\hline \multirow[t]{2}{*}{$36-47$ months } & No & 1.00 & & \\
\hline & Yes & 2.04 & $0.35-11.85$ & 0.429 \\
\hline \multirow[t]{2}{*}{$48-59$ months } & No & 1.00 & & \\
\hline & Yes & 261.97 & $1.46-47281.50$ & 0.036 \\
\hline
\end{tabular}

IMCI - Integrated Management of Childhood Illness, CI - confidence interval, OR - odds ratio

*Mixed-effects logistic regression models quantified the influence of IMCI in-service training on counting respiratory rates for 60 s (or not) across each age group adjusted for variables listed in Table 3 and data clustering. Results for the children aged 48-59 months should be interpreted with caution due to few observations and positive outcomes. 


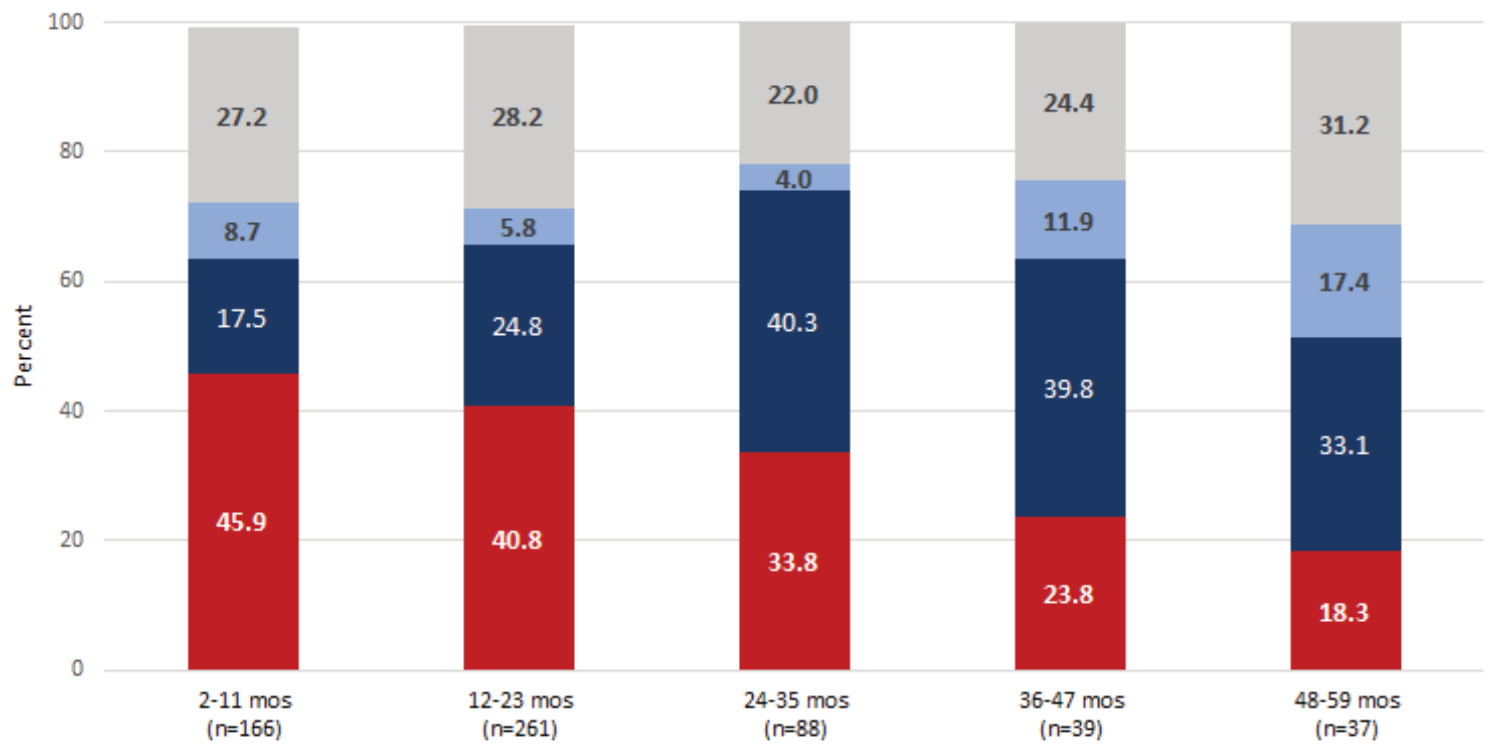

amoxicillin or benzyl penicillin injection

Figure 2. Antibiotic prescriptions for IMCI non-severe pneumonia by age groups, Malawi health facilities, 2013-2014. Frequencies were weighted to account for the unequal probabilities of selection due to differing client volumes on the interview date.

Table 5. Determinants of first-line antibiotic treatment for IMCI non-severe pneumonia in outpatients aged 2-59 month, Malawi health facilities, 2013-2014*

\begin{tabular}{|c|c|c|c|c|c|}
\hline & & & AdJUSted OR & $95 \%$ CI & $\mathbf{P}$ \\
\hline \multirow[t]{8}{*}{ Patient } & Temperature (Celsius) & 37.5 or less & 1.00 & & \\
\hline & & $37.6-38.9$ & 1.17 & $0.69-1.97$ & 0.569 \\
\hline & & $39.0-40.8$ & 3.26 & $1.24-8.55$ & 0.016 \\
\hline & Child's age (months) & $2-11$ & 1.00 & & \\
\hline & & $12-23$ & 0.83 & $0.48-1.43$ & 0.502 \\
\hline & & $24-35$ & 0.62 & $0.31-1.25$ & 0.183 \\
\hline & & $36-47$ & 0.33 & $0.12-0.88$ & 0.027 \\
\hline & & $48-59$ & 0.29 & $0.10-0.83$ & 0.021 \\
\hline \multirow[t]{8}{*}{ Facility } & Transmission season & Peak & 1.00 & & \\
\hline & & Off-peak & 0.61 & $0.29-1.27$ & 0.187 \\
\hline & Region & North & 1.00 & & \\
\hline & & Central & 1.87 & $0.88-3.97$ & 0.103 \\
\hline & & South & 0.94 & $0.45-1.98$ & 0.875 \\
\hline & Total staff doctors & 0 & 1.00 & & \\
\hline & & 1 & 0.58 & $0.18-1.89$ & 0.264 \\
\hline & & 2 or more & 2.90 & $1.22-6.88$ & 0.016 \\
\hline
\end{tabular}

IMCI - Integrated Management of Childhood Illness, OR - odds ratio, CI - confidence interval

*Table S2 in Online Supplementary Document presents descriptive statistics for outpatients aged 2-59 mo with IMCI non-severe pneumonia classification receiving first-line antibiotic treatment $(n=590)$. CI refers to confidence interval. Variables presented in this table were significant $(P<0.1)$ in bivariate analyses and were subsequently included simultaneously in the final model to obtain adjusted odds ratios. Mixed-effects logistic regression models quantified the influence of variables on first-line antibiotic treatment (or not) adjusted for data clustering. A total of 13 observations (unweighted) with IMCI non-severe pneumonia attended facilities without amoxicillin available on the interview date and these observations were removed from this analysis. Analyses for no antibiotic prescription for IMCI non-severe pneumonia found only diarrhea significantly associated with no treatment in the bivariate analyses (crude OR=1.80, 95\% CI: 1.08-3.01). In the subset analysis among sick child clients aged 2-59 mo with IMCI non-severe pneumonia and RDT results ( $\mathrm{n}=216)$, RDT-positive cases had 10.65 times higher odds of no antibiotic prescription than RDT-negative cases (adjusted OR=10.65, 95\% CI: 2.39-47.36). 
In contrast, those with IMCI non-severe pneumonia who also presented with a diarrhea complaint had significantly higher odds of no antibiotic treatment in the bivariate analysis (OR=1.80, 95\% CI: 1.083.01) while no other significant bivariate associations were identified. Among classified cases with RDT results ( $\mathrm{n}=216$ ), RDT-positive cases had significantly higher odds of no antibiotic receipt than RDTnegative cases (95\% CI: 2.39-47.36) although the effect size should be interpreted with caution due to few observations and positive outcomes.

\section{DISCUSSION}

Overall, there was sub-optimal IMCI-pneumonia care in Malawi health facilities in 2013-2014 in terms of completed assessments and antibiotic prescriptions for non-severe cases. Child's symptoms and age, malaria diagnosis and provider training were main influences on assessment and treatment practices in this study, which could help inform IMCI training and support to improve pneumonia care for sick children.

In this study, IMCI non-severe pneumonia classification was significantly associated with raised temperature, child's age and illness duration that are biologically plausible results and consistent with findings from other research $[12,13,28]$. While previous studies have shown strong correlations between measured or reported fever and IMCI-pneumonia classification, our findings indicate that nearly 1 in 5 (17\%) children without a measured fever $\left(37.5^{\circ} \mathrm{C}\right.$ or less) had IMCI non-severe pneumonia classification in re-examination. This result is higher than expected and may potentially reflect rapid breathing over-diagnosis in re-examination since misclassification can occur even among trained providers [29].

Few patients with CDB complaints had a 60-second respiratory rate counted in the observation, which is consistent with research from Malawi and other settings [5,6,8]. Assessment for IMCI-pneumonia occurred less often if other symptoms were reported (fever, diarrhea, skin problems or any danger sign) or for RDT-confirmed malaria cases as well as among older children, those living in the South region or outpatients attending providers without IMCI in-service training or with lower job qualifications. Other research in both African and Asian settings has shown that malaria diagnosis or reporting other symptom complaints reduced correct IMCI-pneumonia management [23,30,31]. It is critical that IMCI training reinforce the importance of assessing outpatients for multiple conditions given symptom overlap and common co-morbidities particularly among the sickest children at highest risk of death [32].

Some evidence also suggests that IMCI training may improve health worker skills reinforcing current findings although the long-term impact of training programs on performance has been debated [33,34]. Our results also indicate poor assessment performance among lower-level providers that has been shown in other research from Malawi, and enhanced IMCI support may need to specifically target these providers [23]. Poorer IMCI-pneumonia assessment in the South region compared to the North has not been previously reported to our knowledge but is consistent with greater child mortality reductions found in the North than the South region in 2000-2013 [15].

There was a significant and consistent decline in counting respiratory rates with increasing age of the child that has been shown in other settings [35], and could reflect more clinical probing of infants given higher mortality rates in this age group. There may also be higher suspicion of bacterial infections in infants that could also drive higher assessment rates as suggested in qualitative research [24]. Yet while children aged 12-23 months are less often assessed for IMCI-pneumonia than infants, our findings indicate higher rates of IMCI non-severe pneumonia classification in this age group. This disconnect should be addressed through enhanced IMCI training and support, particularly since our stratified analysis suggests IMCI training may potentially have greater effect on raising assessment rates in this older age group. Beyond the clinical setting, the identified under-classification and under-treatment of pneumonia cases may also be informative for regional and global burden of disease estimates more broadly [2].

Common mistreatment of IMCI non-severe pneumonia cases was also found in this analysis. Few (39\%) cases were prescribed first-line antibiotics while $27 \%$ classified cases received no antibiotic prescription despite attendance at facilities with available amoxicillin. Poor antibiotic targeting for IMCI non-severe pneumonia closely follows poor assessments for antibiotic need as previously discussed. First-line antibiotic prescription for classified cases was associated with raised temperature, child's age and total staff doctors while receipt of no antibiotic prescription was associated with diarrhea complaint and RDT-confirmed malaria. Importantly, there was a significant and consistent decline in first-line treatment with increasing age of the child that also occurred with IMCI-pneumonia assessment. In addition, among older children with IMCI-pneumonia, second-line treatment (cotrimoxazole or other antibiotic) was more often prescribed even with amoxicillin available in the facility. This could suggest prioritization of first-line treatment for younger children at higher risk of dying or where there may be greater suspicion of bacterial infection. Higher temperatures also seemed to decrease the likelihood of pneumonia assessment while 
increasing the potential for antibiotic prescriptions suggesting sicker children are prioritized for treatment regardless of assessed need.

Results should be viewed in light of some data limitations. First, health workers may perform better during observations and there could be worse IMCI-pneumonia care in routine practice although this effect could wane over repeated observations [36,37]. Second, the observation protocol does not record all assessments in the IMCI pneumonia algorithm such as looking for wheeze, chest in-drawing or stridor that are needed to classify severe pneumonia. The quality of the assessment is also not recorded such as whether breathing rates were correctly counted in a calm child for 60 seconds while using a timer. Third, the re-examination protocol was limited to a 60-second respiratory rate count, measured temperature and recorded signs of anemia given time and staff constraints. Other signs of severe pneumonia were not assessed such as chest in-drawing or hypoxia nor were there assessments of general danger signs indicating severe disease requiring urgent attention. For these reasons, this study could not assess correct IMCIpneumonia management overall including differentiation among severe from non-severe cases, nor the full IMCI protocol more broadly. Also, we did not assess the new IMCI pneumonia algorithm defining non-severe pneumonia as fast breathing and/or chest in-drawing. It is possible that chest in-drawing could improve pneumonia classification although some children with IMCI-pneumonia will not present with in-drawing making current results of continued importance. It is also possible that chest in-drawing assessment could be similarly associated with child's age or other symptoms as found in this study. Fourth, rapid breathing can be difficult to assess even by trained providers leading to misclassification in either direction [29]. Finally, the IMCI algorithm was designed to include deliberate over-treatment of targeted conditions and recent studies have found IMCI-pneumonia is commonly classified in children who do not have x-ray confirmed disease [38,39]. Many outpatients with IMCI-pneumonia in this analysis likely do not need antibiotics although IMCI guidelines specify antibiotic treatment for presence of cough and fast breathing. The current lack of diagnostics to identify those in need of antibiotics will sustain continued misdirected treatment practices.

\section{CONCLUSIONS}

Based on a national facility census including 3149 observed sick children aged 2-59 months, study findings indicate sub-optimal care for IMCI non-severe pneumonia in terms of completed assessments and antibiotic prescriptions. Few classified cases received first-line antibiotics and counting respiratory rates was not often conducted. Results reinforce the primary importance of child's symptoms and age, malaria diagnosis as well as provider qualification and training on IMCI-pneumonia care in Malawi health facilities. Enhanced training and support is needed improve IMCI implementation particularly for the pneumonia algorithm, and current results suggest ways to better target these programs in the future. Greater focus on improved IMCI pneumonia care is urgently needed given its major contribution to child mortality in Malawi and globally.

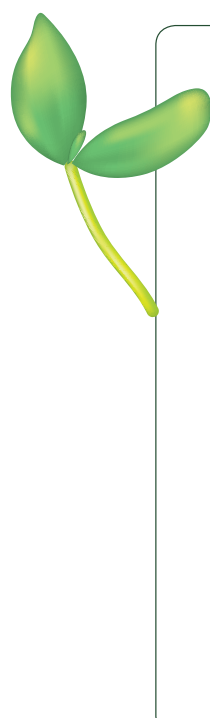

Ethics approval: Our study was a secondary analysis of public datasets. Ethical approval for collection of these data was obtained by the DHS Program from the Department of Health and Human Services Institutional Review Board (IRB) and the host country IRB, which includes authorization to distribute unrestricted survey files for secondary analysis purposes upon receipt of a research proposal. Written informed consent was obtained separately from health workers and caregivers prior to participation in the observation, exit interview and re-examination.

Funding: Our study uses public data sets with no associated cost for secondary analysis. The funders had no role in study design, data collection and analysis, decision to publish, or preparation of the manuscript. EWJ received funding from Uppsala University as a doctoral student. HH was supported by the Stockholm County Council (clinical research appointment). Salary support for HN was from the Malawi Ministry of Health. AA and LC received salary support from the United Nations Children's Fund.

Authorship declarations: EWJ, HH designed and conceptualized the study. EWJ compiled, prepared, analyzed and interpreted data. EWJ, HH, AA contributed to data analysis. EWJ, HN, LCA, AA, HH contributed to interpretation of findings. EWJ wrote the first draft of the paper. EWJ, HN, LCA, AA, HH reviewed, revised and contributed writing to the paper. All authors read and approved the final manuscript.

Competing interests: The authors have completed the Unified Competing Interest form at www.icmje.org/coi_disclosure.pdf (available on request from the corresponding author) and declare no competing interests. 
1 UNICEF. Levels and trends in child mortality report 2015: estimates developed by the UN inter-agency group for child mortality estimation. New York: UNICEF; 2015.

2 Liu L, Oza S, Hogan D, Perin J, Rudan I, Lawn JE, et al. Global, regional and national causes of child mortality in 200013 with projections to inform post-2015 priorities: an updated systematic review. Lancet. 2015;385:430-40. Medline:25280870 doi:10.1016/S0140-6736(14)61698-6

3 Bhutta ZA, Das J, Walker N, Rizvi A, Campbell H, Rudan I, et al. Interventions to address childhood pneumonia and diarrhea equitably: what works and at what cost? Lancet. 2013;381:1417-29. Medline:23582723 doi:10.1016/S01406736(13)60648-0

4 Gove S. Integrated management of childhood illness by outpatient health workers: technical basis and overview. The WHO Working Group on Guidelines for Integrated Management of the Sick Child. Bull World Health Organ. 1997;75 Suppl 1:7-24.

5 Bryce J, Victora C, Habicht JP, Black RE, Scherpbier R. Programmatic pathways to child survival: results of a multi-country evaluation of integrated management of childhood illness. Health Policy Plan. 2005;20:i5-17. Medline:16306070 doi:10.1093/heapol/czi055

6 Chopra M, Binkin NJ, Mason E, Wolfheim C. Integrated management of childhood illness: what have we learned and how can it be improved? Arch Dis Child. 2012;97:350-4. Medline:22278806 doi:10.1136/archdischild-2011-301191

7 Gera T, Shah D, Garner P, Ricahrdson M, Sachdev HS. Integrated management of childhood illness (IMCI) strategy for children under five. Cochrane Database Syst Rev. 2016;6:CD010123. Medline:27378094

8 Bjornstad E, Preidis GA, Lufesi N, Olson D, Kamthunzi P, Hosseinipour MC, et al. Determining the quality of IMCI pneumonia care in Malawian children. Paediatr Int Child Health. 2014;34:29-36. Medline:24091151 doi:10.1179/2046905 513Y.0000000070

9 Enarson PM, Gie RP, Mwansambo CC, Chalira AE, Lufesi NN, Maganga ER, et al. Potentially Modifiable factors associated with death of infants and children with severe pneumonia routinely managed in district hospitals in Malawi. PLoS One. 2015;10:e0133365. Medline:26237222 doi:10.1371/journal.pone.0133365

10 Kalu N, Lufesi N, Havens D, Mortimer K. Implementation of World Health Organization Integrated Management of Childhood Illnesses (IMCI) Guidelines for the assessment of pneumonia in the under 5s in rural Malawi. PLoS One. 2016;11:e0155830. Medline:27187773 doi:10.1371/journal.pone.0155830

11 Government of Malawi, Ministry of Health and ICF International. Malawi Service Provision Assessment 2013-14. Lilongwe, Malawi and Rockville, Maryland, USA: Government of Malawi and ICF International; 2015.

12 Källander K, Nsungwa-Sibiiti J, Peterson S. Symptom overlap for malaria and pneumonia - policy implications for home management strategies. Acta Trop. 2004;90:211-4. Medline:15177148 doi:10.1016/j.actatropica.2003.11.013

13 Bassat Q, Machevo S, O’Callaghan-Gordo C, Sigauque B, Morais L, Diez-Padrisa N, et al. Distinguishing malaria from severe pneumonia among hospitalized children who fulfilled Integrated Management of Childhood Illness criteria for both diseases: a hospital-based study in Mozambique. Am J Trop Med Hyg. 2011;85:626-34. Medline:21976562 doi:10.4269/ajtmh.2011.11-0223

14 United Nations. World population prospects: the 2015 revision. New York: United Nations; 2015.

15 Kanyuka M, Ndwala J, Mleme T, Chisesa L, Makwemba M, Amouzou A, et al. Malawi and Millennium Development Goal 4: a Countdown to 2015 country case study. Lancet Glob Health. 2016;4:e201-14. Medline:26805586 doi:10.1016/ S2214-109X(15)00294-6

16 Government of Malawi, Ministry of Health. Health sector strategic plan 2011-2016. Lilongwe: Government of Malawi; 2011.

17 Government of Malawi, Ministry of Health. Malawi Integrated Management of Childhood Illness. Lilongwe: Government of Malawi; 2013.

$18 \mathrm{WHO}$. Revised WHO classification and treatment for childhood pneumonia at health facilities: evidence summaries. Geneva: WHO; 2014.

19 Rowe AK, Onikpo F, Lama M, Deming MS. The rise and fall of supervision in a project designed to strengthen supervision of integrated management of childhood illness in Benin. Health Policy Plan. 2010;25:125-34. Medline:19923206 doi:10.1093/heapol/czp054

20 Lange S, Mwisongo A, Maestad O. Why don't clinicians adhere more consistently to the guidelines for integrated management of childhood illness? Soc Sci Med. 2014;104:56-63. Medline:24581062 doi:10.1016/j.socscimed.2013.12.020

21 Walter ND, Lyimo T, Skarbinski J, Metta E, Kahigwa E, Flannery B, et al. Why first-level health workers fail to follow guidelines for managing severe disease in children in the coast region, the United Republic of Tanzania. Bull World Health Organ. 2009;87:99-107. Medline:19274361 doi:10.2471/BLT.08.050740

22 Osterholt DM, Onikpo F, Lama M, Deming MS, Rowe AK. Improving pneumonia case management in Benin: a randomized trial of a multi-faceted intervention to support health worker adherence to integrated management of childhood illness guidelines. Hum Resour Health. 2009;7:77. Medline:19712484 doi:10.1186/1478-4491-7-77

23 Steinhardt LC, Chinkhumba J, Wolkon A, Luka M, Luhanga M, Sande J, et al. Patient-, health worker-, and health facility-level determinants of correct malaria case management at publicly funded health facilities in Malawi: results from a nationally representative health facility survey. Malar J. 2014;13:64. Medline:24555546 doi:10.1186/1475-2875-13-64

24 Johansson EW, Kitutu FE, Mayora C, Awor P, Peterson S, Wamani H, et al. 'It could be viral but you don't know, you have not diagnosed it': health worker challenges in managing non-malaria pediatric fevers in the low transmission area of Mbarara District, Uganda. Malar J. 2016;15:197. Medline:27066829 doi:10.1186/s12936-016-1257-y 
25 Johansson EW, Selling KE, Nsona H, Mappin B, Gething PW, Petzold M, et al. Integrated pediatric fever management and antibiotic over-treatment in Malawi health facilities: data mining a national facility census. Malar J. 2016;15:396. Medline:27488343 doi:10.1186/s12936-016-1439-7

26 Bhatt S, Weiss DJ, Cameron E, Bisanzio D, Mappin B, Dalrymple U, et al. The effect of malaria control on Plasmodium falciparum in Africa between 2000 and 2015. Nature. 2015;526:207-11. Medline:26375008 doi:10.1038/nature15535

27 Mapping Malaria Risk in Africa [Internet]. Basel, Switzerland: MARA Database. Available from: http://www.mara-database.org.

$28 \mathrm{WHO}$. The overlap in clinical presentation and treatment of malaria and pneumonia in children: a report of a meeting. Geneva: World Health Organization; 1991.

29 Muro F, Mtove G, Nosha N, Wangai H, Harrison N, Hildenwall H, et al. Effect of context on respiratory rate measurement in identifying non-severe pneumonia in African children. Trop Med Int Health. 2015;20:757-65. Medline:25728867 doi:10.1111/tmi. 12492

30 Senn N, Rarau P, Salib M, Manong D, Siba P, Rogerson S, et al. Use of antibiotics within the IMCI guidelines in outpatient settings in Papua New Guinean children: an observational and effectiveness study. PLoS One. 2014;9:e90990. Medline:24626194 doi:10.1371/journal.pone.0090990

31 Acacio S, Verani JR, Lanaspa M, Fairlie TA, Nhampossa T, Ruperez M, et al. Under treatment of pneumonia among children under 5 years of age in a malaria-endemic area: population-based surveillance study conducted in Manhica district in rural Mozambique. Int J Infect Dis. 2015;36:39-45. Medline:25980619 doi:10.1016/j.jiid.2015.05.010

32 Fischer Walker CL, Perin J, Liu JL, Katsz J, Tielsch JM, Black RE. Does comorbidity increase the risk of mortality among children under 3 years of age? BMJ Open. 2013;3:e003457. Medline:23965935 doi:10.1136/bmjopen-2013-003457

33 Nguyen DTK, Leung K, McIntyre L, Ghali W, Sauve R. Does Integrated Management of Childhood Illness improve the skills of health workers? A systematic review and meta-analysis. PLoS One. 2013;8:e66030. Medline:23776599 doi:10.1371/journal.pone.0066030

34 Pariyo GW, Gouws E, Bryce J, Burnham G. Improving facility-based care for sick children in Uganda: training is not enough. Health Policy Plan. 2005;20:i58-68. Medline:16306071 doi:10.1093/heapol/czi051

35 Steinhardt LC, Onikpo F, Kouame J, Piercefield E, Lama M, Deming MS, et al. Predictors of health worker performance after Integrated Management of Childhood Illness training in Benin: a cohort study. BMC Health Serv Res. 2015;15:276. Medline:26194895 doi:10.1186/s12913-015-0910-4

36 Leonard K, Masatu MC. Outpatient process quality evaluation and the Hawthorne Effect. Soc Sci Med. 2006;63:233040. Medline:16887245 doi:10.1016/j.socscimed.2006.06.003

37 McCambridge J, Witton J, Elbourn D. Systematic review of the Hawthorne effect: new concepts are needed to study research participant effects. J Clin Epidemiol. 2014;67:267-77. Medline:24275499 doi:10.1016/j.jclinepi.2013.08.015

38 Hildenwall H, Amos B, Mtove G, Muro F, Cederlund K, Reyburn H. Causes of non-malarial febrile illness in outpatients in Tanzania. Trop Med Int Health. 2016;21:149-56. Medline:26544671 doi:10.1111/tmi.12635

39 D’Acremont V, Kilowoko M, Kyungu E, Philipina S, Sangu W, Kahama-Maro J, et al. Beyond malaria - causes of fever in outpatient Tanzanian children. N Engl J Med. 2014;370:809-17. Medline:24571753 doi:10.1056/NEJMoal214482 